\title{
Correspondence: Phantom phonon localization in relaxors
}

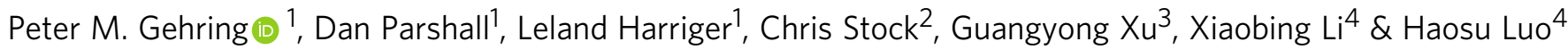

Nature Communications 8:_\#\#\#\# doi:10.1038/s41467-017-01395-6 (2017)

Manley et al. ${ }^{1}$ report the observation of an unexpected, weak, phonon mode located in energy between that of the transverse acoustic (TA) and soft transverse optic (TO) modes in the relaxor ferroelectric $\mathrm{Pb}\left[\left(\mathrm{Mg}_{1 / 3} \mathrm{Nb}_{2 / 3}\right)_{1-x} \mathrm{Ti}_{x}\right] \mathrm{O}_{3}(\mathrm{PMN}-x \mathrm{PT})$ with Ti content $x=0.30$. Referred to as the local mode (LM), the energy of this extra mode varies little with wave vector in the cubic (paraelectric) phase, and this was interpreted as evidence of phonon localization ${ }^{1}$. Any excitation that is localized in space must be extended in reciprocal space $\mathbf{Q}$. To confirm this property, we performed a standard test $\mathrm{t}^{2}$ on a PMN- $x$ PT single crystal of nominally identical composition. This was done by comparing the rates at which the Bragg, TA phonon, and LM neutron scattering cross-sections decrease as the cubic crystallographic [100] axis is tilted out of the horizontal scattering plane by rotating about the orthogonal [010] axis. We find that the LM cross section varies with tilt in a manner identical to that of the Bragg peak. A local mode with short-range spatial correlations that decay exponentially will exhibit a broad Lorentzian peak as a function of tilt. Instead, we observe a narrow Gaussian peak. We conclude that the LM is not a real mode.

We performed a series of identical energy scans at a constant wave vector $\mathbf{Q}=(2,-0.35,0)$ in the cubic phase of a single crystal of PMN-0.29PT as a function of tilt angle (rotation) about the [010] axis. These data were normalized to monitor and corrected for the harmonic content of the incident beam. Following Manley et al. ${ }^{1}$, the TA and TO modes were fit to Lorentzian lineshapes, while the LM was fit to a Gaussian lineshape. The elastic crosssection, which is primarily incoherent in nature because the elastic coherent diffuse scattering is relatively weak in the cubic phase (and at wave vectors far from the zone center), was fit to a Gaussian lineshape. All fits included a constant, flat background.

At zero tilt, we observed the LM at $11.7 \mathrm{meV}$ with a linewidth of $1.8 \mathrm{meV}$ (FWHM) and an energy-integrated intensity $2.7 \%$ times that of the TO mode. These values agree well with those found by Manley et al. ${ }^{1}$ But at tilts of $\pm 6^{\circ}$ the fitted LM intensities are zero: we see no evidence of the LM. To place these results in perspective, the tilt dependences of the LM and TA phonon intensities are plotted in Fig. 1 and normalized to one at zero tilt. The incoherent and the Bragg scattering cross sections are plotted too because they represent opposite extremes in terms of localization.

A truly local mode must exhibit a flat intensity tilt-profile provided the crystal remains fully illuminated by the incident neutron beam. This is exemplified by the elastic incoherent scattering cross section, which changes by $<8 \%$ over the full tilt range. At the opposite extreme is the Bragg cross section, which is a delta function in both $\mathbf{Q}$ and energy; it exhibits a very narrow Gaussian dependence on tilt. The non-zero width reflects the instrumental out-of-plane wave vector resolution. Of these two extremes, both shown in Fig. 1, the LM clearly behaves like a Bragg peak. It varies with tilt far more strongly than does the TA phonon, which is obviously not a local mode. Therefore, the LM is not a local mode.

Because the LM mimics the Bragg cross section, we believe it originates from an elastic-inelastic double-scattering process, as this explains why a small tilt has such a dramatic effect on the LM intensity: tilting rotates the Ewald sphere relative to the crystal reciprocal lattice, thereby breaking the Bragg condition. This concept was used successfully by Rønnow et al. ${ }^{3}$ to explain the spurious "ghoston" peaks seen in $\mathrm{CuGeO}_{3}$. While an elastic-inelastic double-scattering process is weak, Rønnow et al. point out that even modest-sized crystals of order $1 \mathrm{~cm}^{3}$ can exhibit these effects. By comparison, the 200-g crystal used by Manley et al. ${ }^{1}$ corresponds to $25 \mathrm{~cm}^{3}$. In addition, phonon modes in relaxors are much broader than those in conventional perovskites such as $\mathrm{PbTiO}_{3}$; this fact greatly increases the chances of observing ghostons in relaxors because the double-scattering condition is more likely to be satisfied given the larger phonon energy width. Finally, the ghoston model can also explain why Manley et al. ${ }^{1}$ observed the $\mathrm{LM}$ at $\mathbf{Q}=(2,+0.4,0)$ and $(2,-0.4,0)$ : given a ghoston at $(2, q, 0)$ generated by Bragg scattering from

\footnotetext{
${ }^{1}$ NIST Center for Neutron Research, National Institute of Standards and Technology, 100 Bureau Drive, Gaithersburg, MD 20899-600, USA. ${ }^{2}$ School of Astronomy and Physics, University of Edinburgh, Edinburgh EH9 3JZ, UK. ${ }^{3}$ Condensed Matter Physics and Materials Science Department, Brookhaven National Laboratory, Upton, New York, NY 11973, USA. ${ }^{4}$ Shanghai Institute of Ceramics, Chinese Academy of Sciences, Shanghai 201800, China. Correspondence and requests for materials should be addressed to P.M.G. (email: peter.gehring@nist.gov)
} 


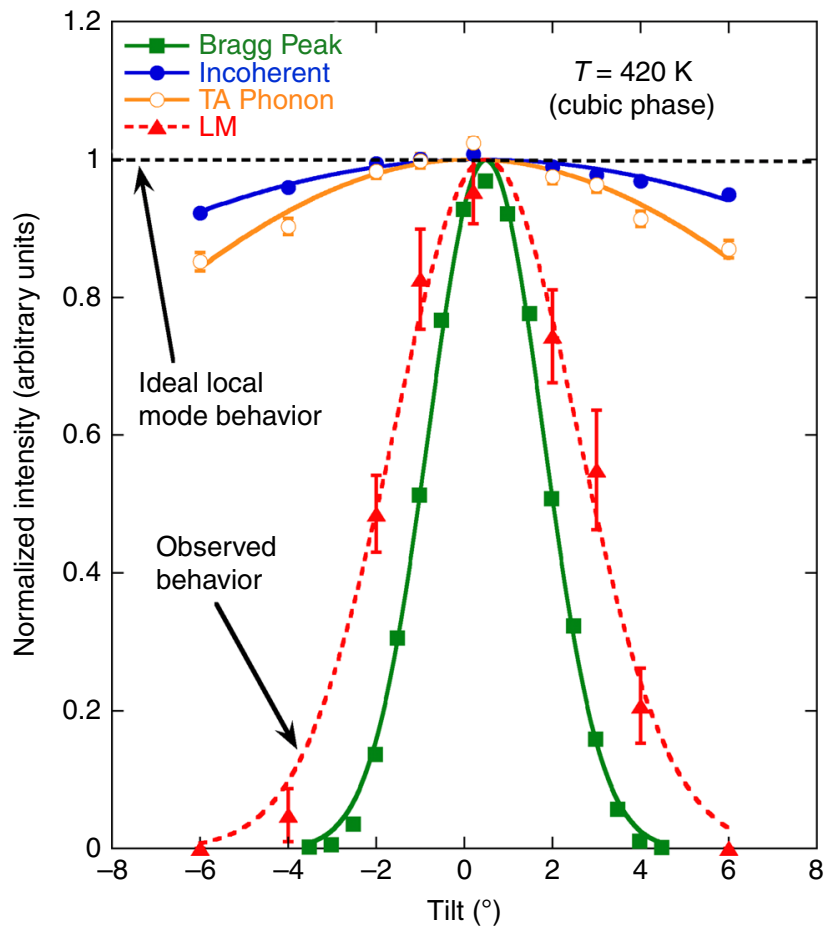

Fig. 1 Tilt dependence of neutron scattering in cubic PMN-0.29PT. The scattering cross sections are obtained at $\mathbf{Q}=(2,-0.35,0)$. LM refers to the feature identified as a local mode by Manley et al. ${ }^{1}$ Lines are fits to Gaussian lineshapes. Errors bars, taken from least-squares fits, denote \pm 1 standard deviation

$\boldsymbol{\tau}=(h, k, l)$, there is always a ghoston of equal energy at $(2,-q, 0)$ generated by Bragg scattering from $(h,-k, l)$.

Our data demonstrate that the LM scattering cross section exhibits an extremely sharp dependence on tilt that is well described by a Gaussian function. These features are inconsistent with those expected for a local mode. We conclude that the central thesis of Manley et al. ${ }^{1}$ is wrong.

\section{Methods}

Triple-axis neutron inelastic scattering. We studied an 80 -g single crystal of PMN- $x$ PT having nominal Ti content $x=0.29$. The crystal was cut with $\{100\}$ faces and dimensions $17.8 \mathrm{~mm} \times 23 \mathrm{~mm} \times 24.3 \mathrm{~mm}$ and oriented in the [100]-[010] scattering plane with [100] parallel to the $17.8 \mathrm{~mm}$ dimension. The crystal was loaded into a closed cycle ${ }^{4} \mathrm{He}$ refrigerator and mounted on the NIST BT4 triple- axis spectrometer configured as in the work by Manley et al. ${ }^{1}$. We calibrated the BT4 wavelength and scattering angle using an alumina standard and aligned the analyzer using vanadium, which is an incoherent scatterer.

Data availability. All relevant data are available from the corresponding author.

Received: 20 December 2016 Accepted: 13 September 2017 Published online: 05 December 2017

\section{References}

1. Manley, M. E. et al. Phonon localization drives polar nanoregions in a relaxor ferroelectric. Nat. Commun. 5, 3683 (2014).

2. Shirane, G., Shapiro, S. M. \& Tranquada, J. M. Neutron Scattering with a TripleAxis Spectrometer (Cambridge University Press, 2002).

3. Rønnow, H. M., Regnault, L. P. \& Lorenzo, J. E. Chasing ghosts in reciprocal space - a novel inelastic neutron multiple scattering process. Physica B $\mathbf{3 5 0}$ $11-16(2004)$

\section{Author contributions}

P.M.G., D.P., C.S., and G.X. conceived of the experiments. P.M.G., D.P., and C.S. performed the triple-axis measurements. P.M.G. analyzed the data. X.L. and H.L. grew the crystal. P.M.G. wrote the manuscript with input from D.P., C.S., L.H., and G.X.

\section{Additional information}

Competing interests: The authors declare no competing financial interests.

Reprints and permission information is available online at http://npg.nature.com/ reprintsandpermissions/

Publisher's note: Springer Nature remains neutral with regard to jurisdictional claims in published maps and institutional affiliations.

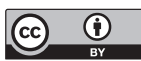

Open Access This article is licensed under a Creative Commons Attribution 4.0 International License, which permits use, sharing, adaptation, distribution and reproduction in any medium or format, as long as you give appropriate credit to the original author(s) and the source, provide a link to the Creative Commons license, and indicate if changes were made. The images or other third party material in this article are included in the article's Creative Commons license, unless indicated otherwise in a credit line to the material. If material is not included in the article's Creative Commons license and your intended use is not permitted by statutory regulation or exceeds the permitted use, you will need to obtain permission directly from the copyright holder. To view a copy of this license, visit http://creativecommons.org/ licenses/by/4.0/.

(C) The Author(s) 2017 